\title{
RF MANIPULATIONS IN THE FERMILAB MAIN INJECTOR*
}

\author{
I. Kourbanis, and D. Wildman, FNAL, Batavia, IL 60510 USA
}

\section{Abstract}

The Fermilab Main Injector will have a multifunctional role in the Run II Collider run. It will not only provide beam for antiproton stacking and intense proton and antiproton bunches to the Tevatron collider, but will also decelerate antiprotons for recycling in the new Recycler Ring [1]. To accomplish these goals a series of RF manipulations will be needed.

\section{PROTON COALESCING}

In order to provide intense proton bunches $\left(270 \times 10^{9} \mathrm{ppb}\right)$ for the Tevatron, five to seven $53 \mathrm{MHz}$ proton bunches will be colalesced using a rotation in a $2.5 \mathrm{MHz}$ harmonic RF system [2],[3]. The coalesced bunch is then recaptured with $1 \mathrm{MV}$ of $53 \mathrm{MHz}$ and then the voltage is adiabatically reduced to $440 \mathrm{KV}$ to match the $1 \mathrm{MV}$ Tevatron voltage. Before coalescing each of the proton bunches has a longitudinal emittance of about $0.15 \mathrm{eV}$-sec and a typical intensity of $40-50 \times 10^{9} \mathrm{p}$. The coalesced bunch will have a longitudinal emittance of 1.4-2.0 eVsec depending on the number of coalesced bunches.

The coalescing process has been simulated with ESME [4]. A mountain range of coalescing is shown in Figure 1.

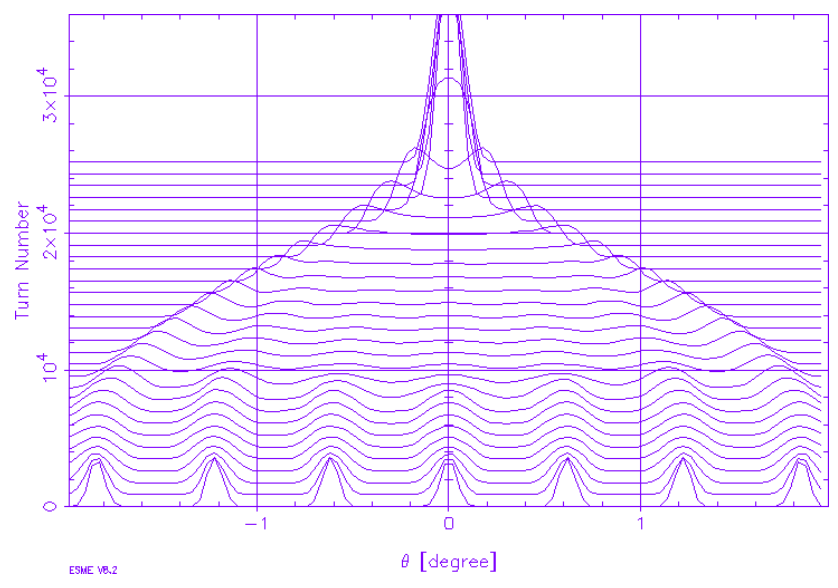

Figure 1: Mountain range picture of the coalescing process. One degree equals $31 \mathrm{nsec}$.

*Operated by Universities Research Association, Inc. under contract with the U.S. Department of Energy.
A total of 36 coalesced proton bunches will be needed in the Tevatron for each store so to avoid repeating the coalescing process 36 times, we plan to eventually inject from the Booster 4 groups of 5-7 bunches each spaced 400 nsec apart accelerate them to $150 \mathrm{GeV}$, and coalesce them. Then the 4 coalesced bunches will be transferred to the Tevatron and the whole process will be repeated 9 times. In order for the multibatch coalescing to work efficiently we need to apply beam loading compensation to the 53 $\mathrm{MHz}$ cavities during the $2.5 \mathrm{MHz}$ rotation.

\section{ANTIPROTON ACCELERATION}

To provide the 36 antiproton bunches for the Tevatron collider, Main Injector will have to accelerate cooled antiproton bunches provided by the Recycler. No antiproton coalescing is needed. The cooled antiproton bunches from the Recycler will have a longitudinal emittance of $1.5 \mathrm{eV}$-sec and intensity of $60 \times 10^{9} \mathrm{p}$ each. Since we are going to exceed the Main Injector momentum aperture if we try to accelerate these large longitudinal emittance bunches trough transition, the 2.5 $\mathrm{MHz}$ coalescing cavities will be used for crossing transition. This will require a $2.5 \mathrm{MHz}$ low level control system with a radial position loop in order to keep the beam in the design radius during the acceleration.

Four antipron bunches at a time will be injected from the Recycler at $8.9 \mathrm{GeV}$ in $2.5 \mathrm{MHz}$ buckets. The $2.5 \mathrm{MHz}$ voltage will be adiabatically raised from $2.0 \mathrm{KV}$ (matching value at injection) to $60 \mathrm{KV}$. Then the four antiproton bunches will be accelerated through transition to $25 \mathrm{GeV}$. At $25 \mathrm{GeV}$ a bunch rotation is performed by dropping the $2.5 \mathrm{MHZ}$ voltage to $6 \mathrm{KV}$ for $1 / 4$ of a synchrotron period and then raising it back to $60 \mathrm{KV}$ for another $1 / 4$ period. After bunch shortening, the antiproton bunches are recaptured in the $53 \mathrm{MHz}$ rf buckets, accelerated to $150 \mathrm{GeV}$, and then transferred into the Tevatron. This entire process is summarized in Fig. 2. The entire acceleration process of one antiproton bunch has been simulated using ESME. A mountain range of the transfer from $2.5 \mathrm{MHz}$ to $53 \mathrm{MHz}$ at $25 \mathrm{GeV}$ is shown in Fig. 3. 


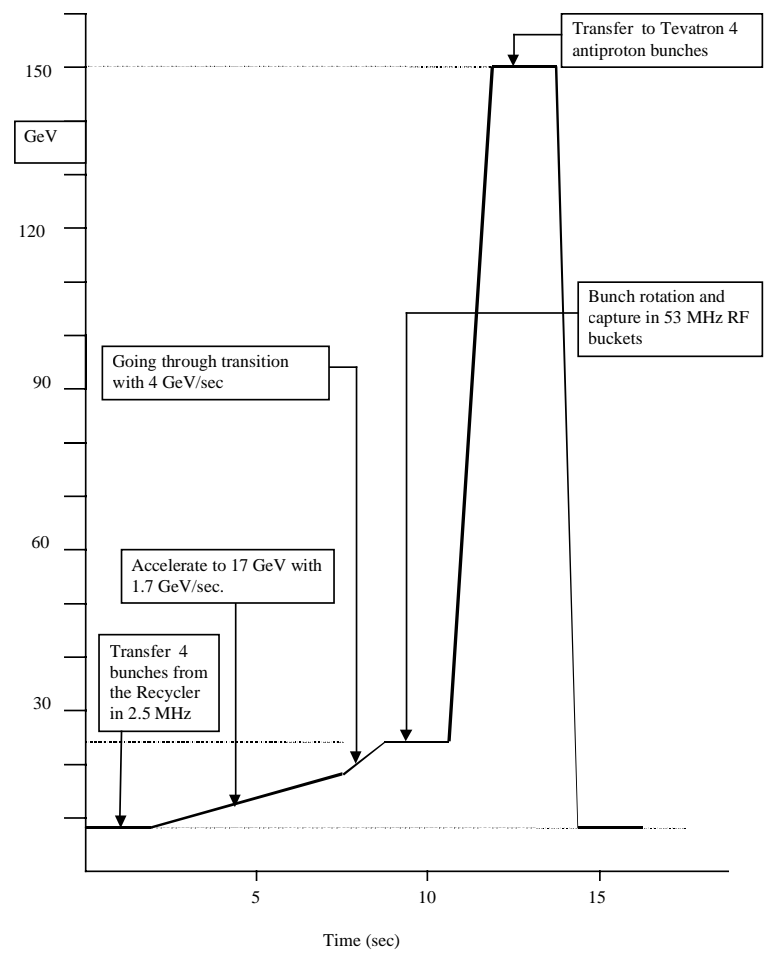

Figure 2: Acceleration of antiproton bunches from the Recycler into the Main Injector.

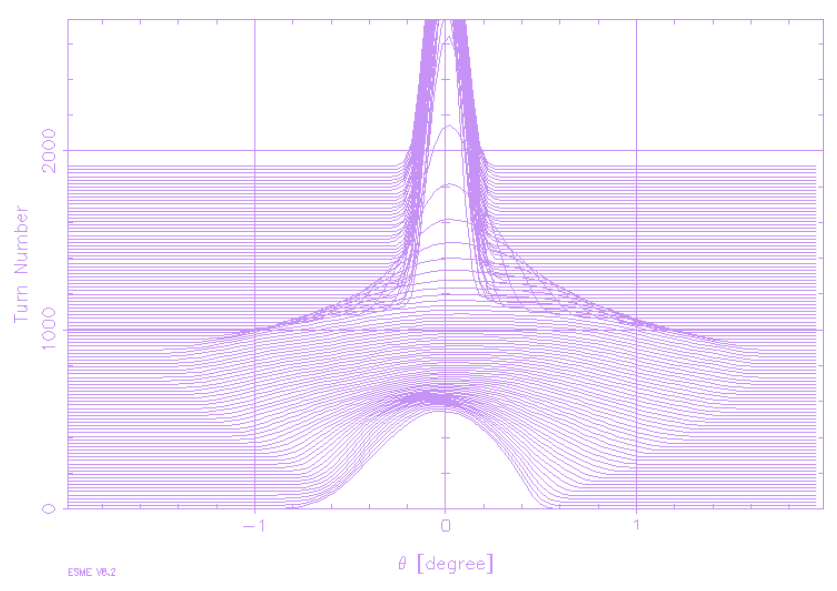

Figure 3: Mountain range picture of the transfer from 2.5 $\mathrm{MHz}$ to $53 \mathrm{MHz}$ at $25 \mathrm{GeV}$ during the antiproton acceleration.

No emittance blowup is observed during the transition crossing. The final longitudinal emittance is $1.65 \mathrm{eV}$-sec with the $10 \%$ growth happening during the transfer from the 2.5 to $53 \mathrm{MHz}$. Beam loading on the $2.5 \mathrm{MHz}$ cavities is a concern that needs to be addressed.

\section{ANTIPROTON DECELERATION}

The antiproton bunches left at the end of the store in the Tevatron collider will be recycled. For this, after the proton bunches are eliminated at $1000 \mathrm{GeV}$, the antiproton bunches will be decelerated to $150 \mathrm{GeV}$. From there they will be transferred to the Main Injector four bunches at a time for a total of nine transfers.

The decelerated antiproton bunches will have a typical longitudinal emittances between 3-4 eV-sec so, as with the antiproton acceleration, we will need to transfer to the 2.5 MHz system before going through transition.

The transfer from $53 \mathrm{MHz}$ to $2.5 \mathrm{MHz}$ is accomplished at a front porch at $25 \mathrm{GeV}$. First the $53 \mathrm{MHz}$ voltage is reduced until the beam fills the bucket. The $53 \mathrm{MHz}$ is turned off and the bunches are rotated for a quarter of a period in $2.5 \mathrm{MHz}$ buckets with $60 \mathrm{KV}$ of $2.5 \mathrm{MHz}$ and 12.0 KV of 5.0 MHz. The rotated bunches are captured with $300 \mathrm{~V}$ of $2.5 \mathrm{MHz}$ and then the voltage is adiabatically raised to $60 \mathrm{KV}$. Next the bunches are decelerated through transition to $8.9 \mathrm{GeV}$, the voltage is reduced till the beam fills the bucket and the bunches are transferred to the Recycler. This process is summarized in fig. 4.

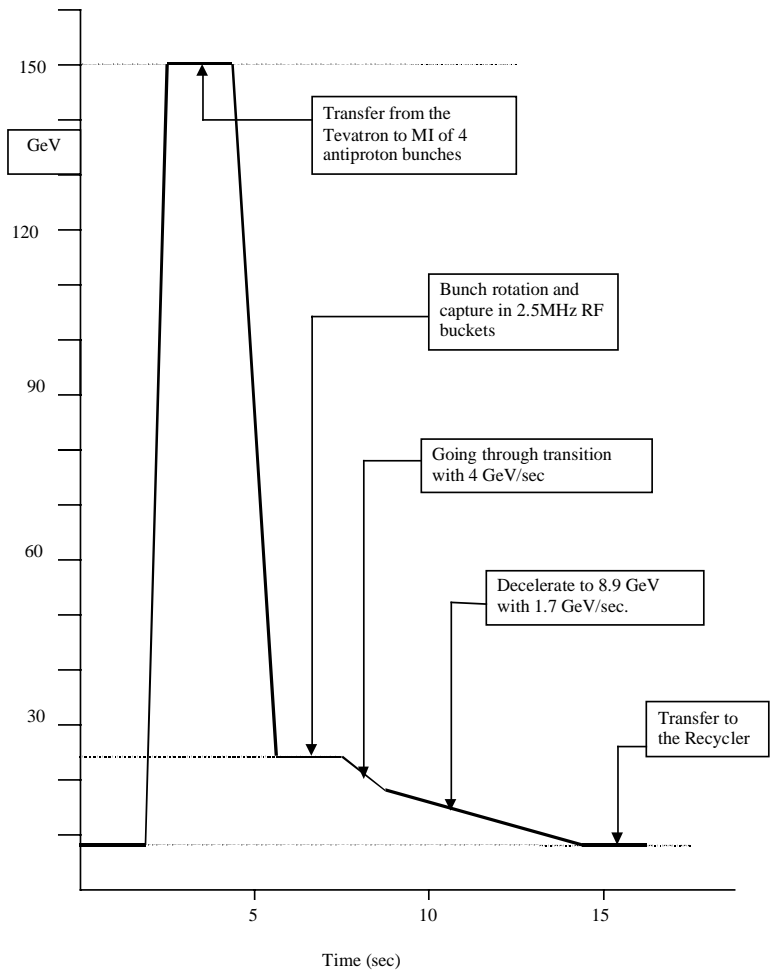

Figure 4: Deceleration process of antiproton bunches in Main Injector. 


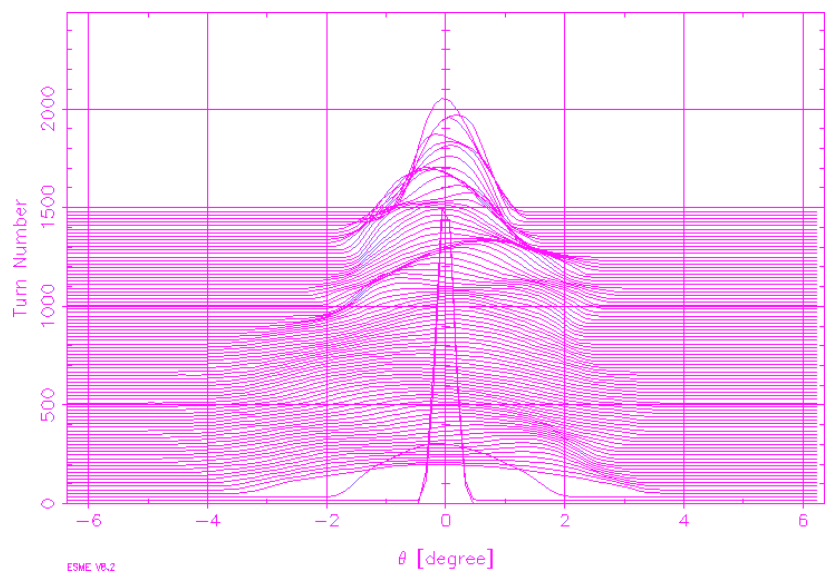

Figure 5: Mountain range picture of the transfer from 53 $\mathrm{MHz}$ to $2.5 \mathrm{MHz}$ at $25 \mathrm{GeV}$ during the antiproton deceleration.

The transition crossing with $4 \mathrm{eV}$-sec bunches results in a $25 \%$ emittance growth, mostly due to nonlinearities because of the slow transition crossing (the nonlinear time $T_{n l}$ much longer than the non-adiabatic time).

To reduce the emittance growth during transition crossing, a bipolar $\gamma_{t}$ jump was considered in the simulations. The bipolar jump considered maintains a clearance of :

$\left[\gamma-\gamma_{t}\right] \geq 0.65 \approx 2 \frac{d \gamma}{d t} T_{n l}$ except for about $10 \mathrm{msec}$.

Transition is crossed at about $d\left(\gamma-\gamma_{t}\right) / d t=130 \mathrm{sec}^{-1}$, almost 30 times faster than wthout a jump. ESME simulations predict that crossing transition with such a $\gamma_{t}$ jump leads to no particle loss and reduces the emittance growth to less than $10 \%$. At present there are no plans for a $\gamma_{t}$ jump during deceleration.

\section{BUNCH ROTATION FOR ANTIPROTON PRODUCTION}

At Fermilab antiprotons are produced by the delivery of trains of $120 \mathrm{GeV}$ proton bunches to a production target from which antiprotons are collected with mean kinetic energy $8 \mathrm{GeV}$ and momentum spread $\Delta p / p \geq 3 \%$. The antiproton beam has the same time structure as the incident protons. The proton bunch spacing-to-length ratio is made as large as possible ( $\geq 20: 1)$ so that the resulting antiproton momentum spread may be reduced by bunch rotation in the debuncher ring where time spread is exchanged for momentum spread.

The narrow bunches in the Main Injector are obtained by doing two successive one-quarter period phase rotations within unmatched buckets. At $120 \mathrm{GeV}$ flattop the rf voltage is held at 3.5 MV so the proton bunches with $0.15 \mathrm{eV}$-sec longitudinal emittance have a full width of about $1.8 \mathrm{nsec}$. The rf voltage is then dropped to $350 \mathrm{KV}$ within about $40 \mu \mathrm{sec}$ by doing a fast paraphasing. Ideally in one quarter synchrotron period ( $4 \mathrm{msec})$ the mismatched bunch rotates to span $5.7 \mathrm{nsec}$ within the bucket. At this point the rf voltage is suddenly raised back to $3.5 \mathrm{MV}$ and the bunch rotates another quarter period so that the bunch energy spread becomes $205 \mathrm{MeV}$ and the full bunch length becomes $0.8 \mathrm{nsec}$.

An ESME picture of the proton bunch narrowing is shown in Fig. 6.

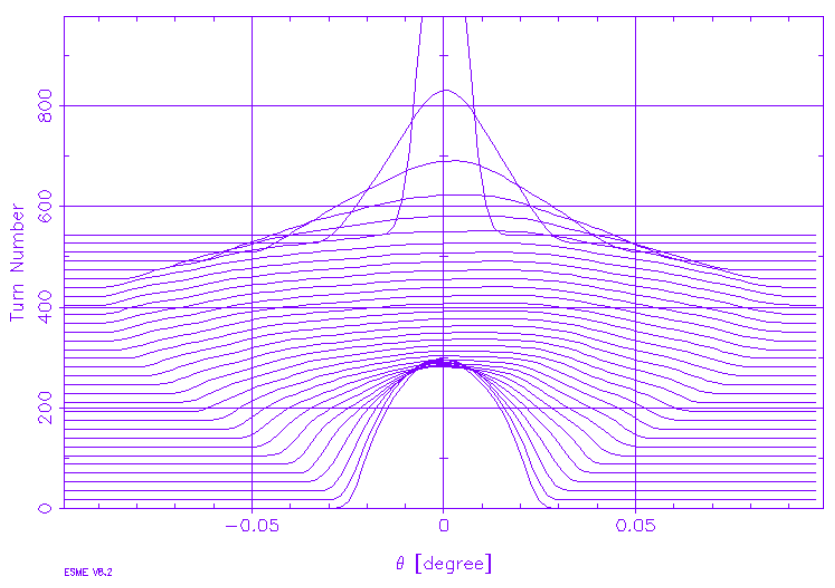

Figure 6: Mountain range picture of the proton bunch narrowing. After two successive bunch rotations the bunch length is reduced by half.

\section{REFERENCES}

[1] G. Jackson, "The Fermilab Recycler Ring Technical Design Report", FERMILAB-TM-1991, (1996)

[2] J. Dey, I. Kourbanis, and D. Wildman "A New RF System For Bunch Coalescing In The Fermilab Main Ring", Proc. of the 1995 Particle Accelerator Conf. , Dallas, TX., May 1-5, 1995, pp. 16721674.

[3] J. Dey, I. Kourbanis, and D. Wildman "Improvements in Bunch Coalescing in the Fermilab Main Ring”, Proc. of the 1995 Particle Accelerator Conf., Dallas, TX., May 1-5, 1995, pp. 3312-3314.

[4] J. A. MacLachlan, Jean-Francois Ostiguy “User's Guide to ESME v. 8.2" September 22, 1997, Unpublished. 